\title{
Some Computer-Assisted Topological Models of Hilbert Fundamental Domains*
}

\author{
By Harvey Cohn
}

\begin{abstract}
The Hilbert modular group $\mathbf{H}$ for the integral domain $\mathbf{O}\left(k^{1 / 2}\right)$ has a fourdimensional fundamental domain $\mathbf{R}$ which should be represented geometrically (like the classic modular group). Computer assistance (by the Argonne CDC 3600) was used for outlining cross sections of the three-dimensional "floor" of $\mathbf{R}$, which is a mosaic of an intractably large number of boundary pieces identified under $H$. The cross sections shown here might well contain enough information when $k=2,3,5,6$ to form some "incidence matrices" and see $\mathbf{R}$ (at least) combinatorially. For special symmetrized subgroups of $\mathbf{H}$, it is plausible to see homologously independent 2-spheres in (the corresponding) $\mathbf{R}$. The program is a continuation of one outlined in two earlier issues of this journal v. 19, 1965, pp. 594-605, MR 33 \#4016, and v. 21, 1967, pp. 76-86, .IR 36 \#.5081.
\end{abstract}

14. Final Program. Here we present the third and final revision of a program aimed at using the computer to help visualize the four-dimensional, fundamental domain of the Hilbert modular group in two complex variables. The improvement on the earlier program in [12] leads to a visualization for the first time of some of the fundamental domains as they might be described topologically, (or rather combinatorially, i.e., without any claim of knowing all or any of the usual algebraic invariants). The groups relate to $\mathbf{O}\left(k^{1 / 2}\right)$ for $k=2,3,5,6$. Some 2 -spheres also are indicated in Section 18 below which (conjecturally) may represent independent homology classes. Special attention will also be given to cases cited by Gundlach [8] where superdomains are compact complex manifolds.

From the computer's point of view only a slight improvement is made over the revised program in [12]. It consists of tagging all of the transformations which occur in (2.5) or (9.4) in the order of their appearance. Thus instead of output diagrams in [12] like Figs. 2, 3, 4 (which are labelled according to the "norm," i.e., $|N(\gamma)|$ ), we shall have diagrams like Fig. 5 where the labelling is fine enough to distinguish different transformations (with possibly the same norm).

Illustratively, Fig. jo represents the cross section $S_{1}=1$ for the case $k=2$, where $R_{1}$ (labelled " $R$ ") and $R_{2}$ (labelled " $R$ PRIME") go from -.50 to +.50 in steps of .05 each, giving a grid of 21 by 21 points spanning the projection of the floor. (We shall temporarily ignore the free-hand markings and designations which are explained in Section 17 below and concentrate only on the numbers printed on the

Received October 30, 1968.

* Presented July 1968 at the Oberwolfach Tagung über Automorphe Funktionen zu arithmetisch definierten Gruppen (spezielle Modulfunktionen). Research was supported by NSF Grant P-6423. Computer Support on the Argonne CDC 3600 was continued through the cooperation of Dr. Wallace J. Givens, Director Applied Mathematics Division of the Argonne National Laboratories, Argonne, Illinois, with Mr. Burton S. Garbow as programmer.

This paper is a continuation of items [6] containing Section 1-Section 8 and [12] containing Section 9-Section 13 (see bibliography) with figures and tables also numbered consecutively. Symbols are explained in Section 2 or Section 3 of [6] and Section 9 of [12] unless other references: are given. 


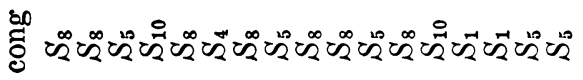

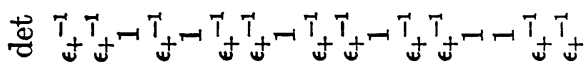

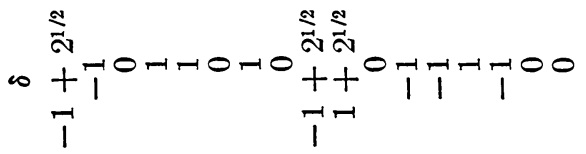

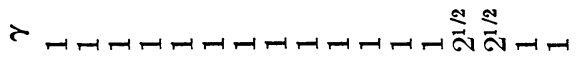

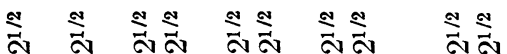

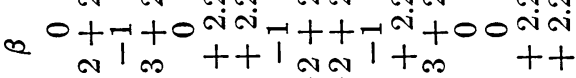
i

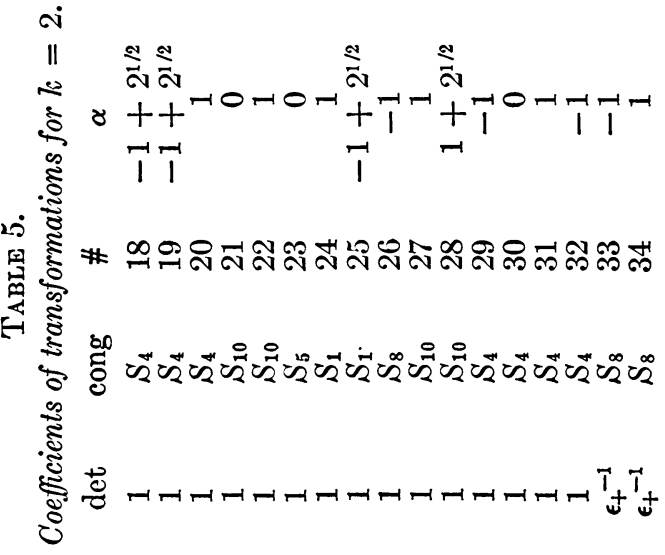

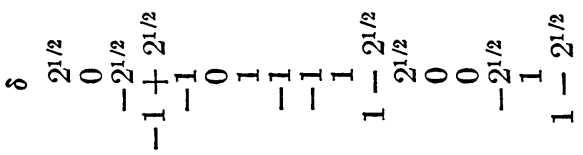

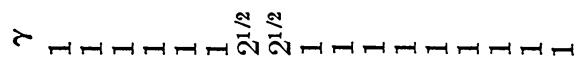
a \% $00000 \frac{1}{1-4}$

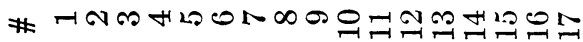


grid-points.) These numbers stand for transformations (9.4) which match points of the floor of the fundamental domain. Indeed in Table 5, the values of $\alpha, \beta, \gamma, \delta$ are listed for the 34 transformations which occur in the range

$$
-1 / 2 \leqq R_{1}, \quad R_{2} \leqq+1 / 2,0 \leqq S_{1} \leqq 1.00 .
$$

(See Remarks (h) and (i) in Section 19 below.) The determinant of the transformation, $\alpha \delta-\beta \gamma$, is also listed (together with congruence classes to be explained in Section 18 below).

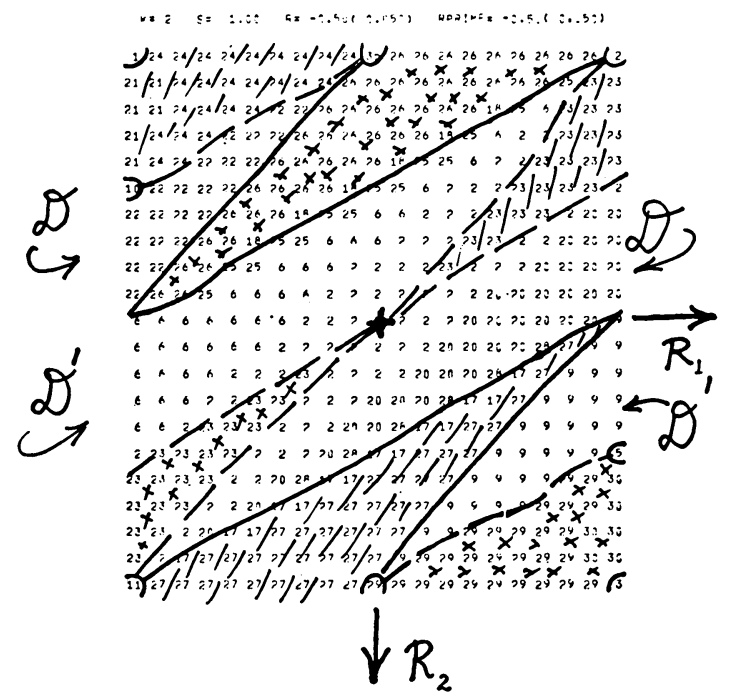

Figure 5. Cross section $S_{1}=1$ for $k=2$.

15. Specification of Subdomains. In order to make the visualization process less formidable, we shall consider supergroups of the Hilbert modular group $\mathbf{H}$ (see (2.5)) defined by symmetry operations, and we shall consider the corresponding subdomain of the fundamental domain $\mathbf{R}$. The motivation is treated elsewhere (see [8], [13]) so let us just remark that the object is to make the point at $\infty$ "compactifiable to an ordinary point" of the manifold.

We consider two ways of augmenting $\mathbf{H}$ by symmetry:

$$
\begin{aligned}
& \mathbf{H}^{+}=\text {augmentation of } \mathbf{H} \text { by } T_{+}\left(Z, Z^{\prime}\right)=\left(Z^{\prime}, Z\right), \\
& \mathbf{H}^{-}=\text {augmentation of } \mathbf{H} \text { by } T_{-}\left(Z, Z^{\prime}\right)=\left(-\tilde{Z}^{\prime},-\tilde{Z}\right),
\end{aligned}
$$

with reference to the complex conjugate. Of course $T_{+}$and $T_{-}$are considered elements of $\mathbf{H}_{\infty}{ }^{+}, \mathbf{H}_{\infty}{ }^{-}$, the subgroups leaving the point at $\infty$ invariant. (Compare $\mathrm{H}_{\infty}$ in (2.7) above.)

In detail,

$$
\begin{aligned}
& T_{+}\left(X+i Y, X^{\prime}+i Y^{\prime}\right)=\left(X^{\prime}+i Y^{\prime}, X+i Y\right), \\
& T_{-}\left(X+i Y, X^{\prime}+i Y^{\prime}\right)=\left(-X^{\prime}+i Y^{\prime},-X+i Y\right),
\end{aligned}
$$

so that all transformations of $\mathbf{H}, \mathbf{H}^{+}, \mathbf{H}^{-}$leave $\mathrm{U} \times \mathbf{U}$ invariant (see (2.2) above). 
Clearly $\mathrm{H}^{+}$and $\mathrm{H}^{-}$are supergroups in which $\mathrm{H}$ has index 2 .

As usual, we shall denote a transformation by referring to a single variable, and the conjugate will be understood, e.g., if $T(Z)=\left(3+2 \cdot 2^{1 / 2}\right) Z^{\prime}$, then $T\left(Z^{\prime}\right)=$ $\left(3-2 \cdot 2^{1 / 2}\right) Z$, etc.

The fundamental domain of $\mathbf{U} \times \mathbf{U} / \mathbf{H}_{\infty}$ was described in Section 12 (above) as a certain fibred 3-space suspended conically at $\infty$. It is desirable that $\mathbf{U} \times \mathbf{U} / \mathbf{H}_{\infty}{ }^{ \pm}$ should be a 3 -sphere suspended conically at $\infty$, (so that $\infty$ is an ordinary manifold point under the usual compactification).

More specifically, (compare [13]), the fundamental domain of $\mathbf{U} \times \mathbf{U} / \mathbf{H}_{\infty} \pm$ is represented by

$$
\left.R_{1}, R_{2} \text { (modulo } 1\right), \quad 0 \leqq S_{1} \leqq h, \quad 0<S_{2},
$$

subject to further identification on the top surface $S_{1}=h$ and the bottom surface $S_{1}=0$. If we combine (12.2) with (15.3) we obtain at $S_{1}=h$,

$$
T_{ \pm}\left(\begin{array}{l}
R_{1} \\
R_{2}
\end{array}\right)= \pm\left(\begin{array}{ll}
u_{1} & k u_{2} \\
-u_{2} & -u_{1}
\end{array}\right)\left(\begin{array}{l}
R_{1} \\
R_{2}
\end{array}\right)
$$

and, at $S_{1}=0$,

$$
T_{ \pm}\left(\begin{array}{l}
R_{1} \\
R_{2}
\end{array}\right)= \pm\left(\begin{array}{rr}
1 & 0 \\
0 & -1
\end{array}\right)\left(\begin{array}{l}
R_{1} \\
R_{2}
\end{array}\right)
$$

Thus we have the cone at $\propto$ suspended from this "half-cube" with boundary involutions at top and bottom surfaces $\left(S_{1}=h\right.$ and $\left.S_{1}=0\right)$ superimposed on "torus" identifications (modulo 1). These involutions have symmetries at $S_{1}=h$ and $S_{1}=0$ (compare [13]). There is an invariant form $P$ in $R_{1}$ and $R_{2}$ preserved under $T_{+}$(or $T_{-}$) and an anti-invariant form $P^{\prime}$ negated under $T_{+}$(or $T_{-}$). For instance, from (15.5a), these forms are as follows at $S_{1}=h$ :

$$
\begin{aligned}
T_{+}\left(P, P^{\prime}\right) & =\left(P,-P^{\prime}\right), & & \\
P & =\left[\left(u_{1}+1\right) R_{1}+k u_{2} R_{2}\right] / d_{1}, & & d_{1}=\text { g.c.d. }\left(u_{1}+1, k u_{2}\right), \\
P^{\prime} & =\left[\left(u_{1}-1\right) R_{1}+k u_{2} R_{2}\right] / d_{2}, & & d_{2}=\text { g.c.d. }\left(u_{1}-1, k u_{2}\right),
\end{aligned}
$$

while

$$
\begin{array}{rlrl}
T_{-}\left(P, P^{\prime}\right) & =\left(P,-P^{\prime}\right), & \\
P^{\prime} & =\left[\left(u_{1}-1\right) R_{1}+k u_{2} R_{2}\right] / d_{3}, & & d_{3}=\text { g.c.d. }\left(u_{1}-1, k u_{2}\right), \\
P^{\prime} & =\left[\left(u_{1}+1\right) R_{1}+k u_{2} R_{2}\right] / d_{4}, & & d_{4}=\text { g.c.d. }\left(u_{1}+1, k u_{2}\right) .
\end{array}
$$

We temporarily use the same notation $X=R_{1}+R_{2} k^{1 / 2}, X^{\prime}=R_{1}-R_{2} k^{1 / 2}$ regardless of whether $k \equiv 1(\bmod 4)$, in order to simplify the notation in the above equations.

If we consider the most immediate cases we have the following table,

$\begin{array}{llll}k & u_{1} & u_{2} & h \\ 2 & 3 & 2 & 1 \\ 3 & 2 & 1 & 1 \\ 5 & \frac{3}{2} & \frac{1}{2} & 1 \\ 6 & 5 & 2 & 2\end{array}$


For $k=2$, the situation is depicted in Fig. 6 (if we ignore the spindle shaped solids for now). Here, from (15.6a), $P=R_{1}+R_{2}$ and $P^{\prime}=R_{1}+2 R_{2}$ are shown as axes and directions of reflection, at $S_{1}=1$.

According to a study, presented in [13, p. 12], the fundamental domain $\mathrm{U} \times$ $\mathrm{U} / \mathrm{H}_{\infty} \pm$ is a 3-sphere $\left(S^{3}\right)$ projected at $\infty$ (or a manifold at $\infty$ ) precisely when one of the following holds:

$$
T_{+}:\left(u_{1}-1\right) \mid u_{2}
$$

or

$$
T_{-}: u_{2} \mid\left(u_{1}+1\right) \text {. }
$$

It can be shown that the only units for which the condition (15.7a) can be satisfied are $3+2 \cdot 2^{1 / 2}, 2+3^{1 / 2}$, and $\frac{1}{2}\left(3+5^{1 / 2}\right)$. (This follows from the fact that "generally" $u_{1} \approx u_{2} k^{1 / 2}$ so $u_{1}$ is "much larger than" $u_{2}$.) Thus except in the three cases cited $\mathrm{U} \times \mathrm{U} / \mathrm{H}_{\infty}{ }^{+}$can not be a manifold at $\infty$ (under the usual compactification). Of course $\mathrm{U} \times \mathbf{U} / \mathbf{H}_{\infty}{ }^{-}$, on the other hand, can more "easily" be a manifold at $\propto$, as is the case for $5+2 \cdot 6^{1 / 2}, 7+4 \cdot 3^{1 / 2},\left(=\epsilon_{+}{ }^{2}\right.$, compare Section 18 below $)$.

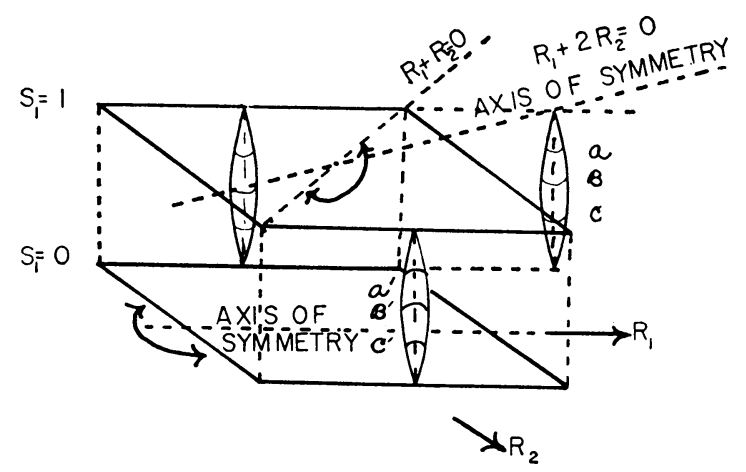

Figure 6. Coordinates of floor for $k=2,\left(T_{+}\right.$symmetry $)$.

16. Assembling the Cross Sections. We now construct the floor of $U \times U / \mathbf{H}$ as lying over the cube $\mathbf{C}$

$$
\text { C }\left\{\begin{array}{l}
-1 / 2 \leqq R_{1} \leqq+1 / 2, \\
-1 / 2 \leqq R_{2} \leqq+1 / 2, \\
-h \leqq S_{1} \leqq+h .
\end{array}\right.
$$

We temporarily disregard boundary identifications to determine the point $\left(R_{1}, R_{2}\right.$, $\left.S_{1}, S_{2}\right)$, i.e., the value of $S_{2}$ lying over each point $\left(R_{1}, R_{2}, S_{1}\right)$, in (say) a grid $\left(S_{1}=\right.$ const) spanning $\mathbf{C}$, (see Section 2 ). Then in terms of the coordinates $Z, Z^{\prime}$, we obtain

$$
\min \|\gamma Z+\delta\|^{2}=1,
$$

with $(\gamma, \delta)$ a pair of integers of $O,(\gamma \neq 0)$ and, as usual,

$$
\|\gamma Z+\delta\|=|\gamma Z+\delta|\left|\gamma^{\prime} Z^{\prime}+\delta^{\prime}\right| .
$$


The optimizing value of $S_{2}$ determines the $\left(Z, Z^{\prime}\right)$ which lies on the floor over $\left(R_{1}, R_{2}, S_{1}\right)$. We also determine by the revised computation [12] a pair $\alpha, \beta \in \mathrm{O}$ such that for some element of $\mathbf{H}$, another point, namely

$$
\begin{aligned}
& Z_{*}=(\alpha Z+\beta) /(\gamma Z+\delta), \\
& Z_{*}{ }^{\prime}=\left(\alpha^{\prime} Z^{\prime}+\beta^{\prime}\right) /\left(\gamma^{\prime} Z^{\prime}+\delta^{\prime}\right),
\end{aligned}
$$

is obtained also lying on the floor over $\mathrm{C}$. The computer labels each point of the cross section according to the transformation obtained.

Now $\mathbf{C}$ is divided into three-dimensional regions in which some one definite transformation (16.4) is valid. (For $k=2$ there are 34 of these transformations listed in Table 5.) What we want to do is to cut and paste these regions to produce a minimum number of them. For example, any region can be "moved" modulo $\mathbf{O}$ or by multiplication by a unit.

There is one limitation, however, which is inherent in the construction, namely the "norm." Let us call

$$
N m(Z)=\left|\gamma \gamma^{\prime}\right|
$$

from (16.4). Thus, easily, $N m(Z)=N m\left(Z^{\prime}\right)=N m\left(Z_{*}\right)=N m\left(Z_{*}{ }^{\prime}\right)$. The norm is unchanged under the "cutting and pasting." Thus there are at least as many pieces as norms. Putting the transformations of $\mathrm{H}$ in matrix form, we note

Lemma 16.1. Let

$$
T=\left(\begin{array}{ll}
\alpha & \beta \\
\gamma & \delta
\end{array}\right) \text { and } T_{0}=\left(\begin{array}{cc}
\alpha_{0} & \beta_{0} \\
\gamma & \delta
\end{array}\right)
$$

be two transformations in $\mathbf{H}$, then for some integral $t$ and $\lambda \in \mathrm{O}$, we can relate the linear fractional transformations by

$$
T(Z)=T_{0}(Z) \epsilon_{+}{ }^{t}+\lambda .
$$

Consider the cutting and pasting process shown in Fig. 5. We see near the center regions " 2 " and " 20 " are treated as one. Certainly, from Table 5 , we have

$$
\Sigma_{2}=\left(\begin{array}{rr}
0 & -1 \\
1 & 0
\end{array}\right) \quad \text { and } \Sigma_{20}=\left(\begin{array}{rr}
1 & -1 \\
1 & 0
\end{array}\right)
$$

so by Lemma 16.1, we expect to find $\Sigma_{20}(Z)=\Sigma_{2}(Z)+1$. Furthermore, comparing extreme left and right, region " 20 " is contiguous with region " 22 ," i.e., $\Sigma_{20}(Z+1)$ $=\Sigma_{22}(Z)$. We now draw (free-hand) lines to delineate regions which are essentially separate. For this we use the heavy line in Fig. 5, but the dotted line separates regions with the same denominator while $t \neq 1$ in (16.6). Thus comparing "2" and " 23 " we see $\Sigma_{23}(Z)=\epsilon_{+} \Sigma_{2}(Z)$. (The shading helps separate such regions and will be explained in Section 17 below.) By assembling the pieces we go from Fig. 5 to the left-hand corner parallelogram in Fig. $7,\left(S_{1}=1\right)$.

The "cutting" and "pasting," however successful, seems somewhat arbitrary. To see a more general treatment consider an earlier concept [12, Section 11] of denominator class of a transformation $Z_{*}=(\alpha Z+\beta) /(\gamma Z+\delta)$, namely the congruence class

$$
-\delta / \gamma(\bmod O)
$$


We subdivide the cube $\mathbf{C}$ (see (16.1)) into open pieces $\mathbf{C}_{j}$ such that the transformation $T_{j}$ will bring a piece $\mathbf{C}_{j}$ into another piece $\mathbf{C}_{l}$, (possibly $l=j$ ), also in $\mathbf{C}$. Each transformation determines a denominator class $-\delta_{j} / \gamma_{j}$ but several (pieces and) transformations may have the same class. Incidentally, since the inverse is $Z=$ $\left(-\delta Z_{*}+\beta\right) /\left(\gamma Z_{*}-\alpha\right)$, the denominator class of the inverse of $T_{j}$ namely $T_{l}$ is $+\alpha_{j} / \gamma_{j}$, fixed by $\alpha_{j} \delta_{j} \equiv-1 \bmod \gamma_{j}$.

Lemma 16.2. Consider all (pieces and) transformations belonging to the denominator class $-\delta_{j} / \gamma_{j}$. Let them have various determinants $\epsilon_{j}$ all congruent to one another modulo $\gamma_{j}$. Then all these pieces can be relocated under $\mathrm{H}_{\infty}$ so that they are matched by $a$ single transformation $T_{j}$.

The proof consists of changing the variable $Z$ in each transformator so that the determinants of the $T_{j}$ are the same. Then the denominators still belong to the same class and a mere translation in (16.6) will accomplish the objective.

Call the new composite pieces "congruence class pieces." Each such "piece" need not be connected, a priori, in the three-dimensional sense (although in practice, so far, their closures seem to be connected). The boundaries must confront pieces of different congruence classes, so the boundaries are made up of pieces of "surface"

$$
\left\|\gamma_{j} Z+\delta_{j}\right\|=\left\|\gamma_{l}+\delta_{l}\right\|=1,
$$

two-dimensional in nature.

Each congruence class piece will match only another (possibly the same) congruence class piece under the transformations which map pieces of the floor onto one another.
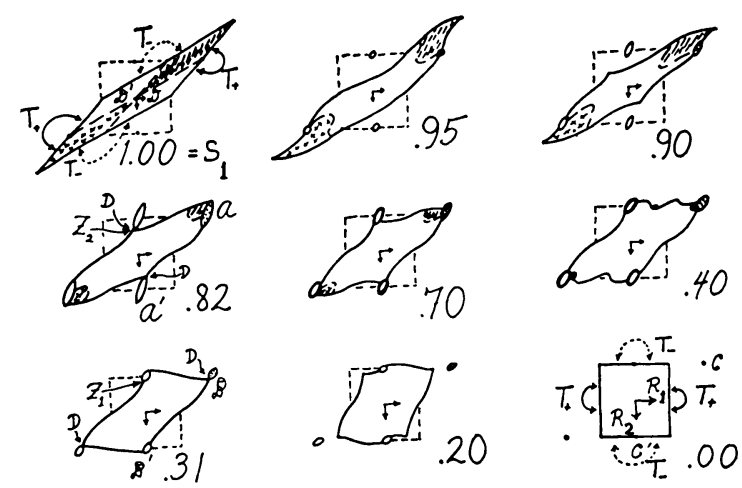

Figure 7. Cross sections of floor for $k=2$.
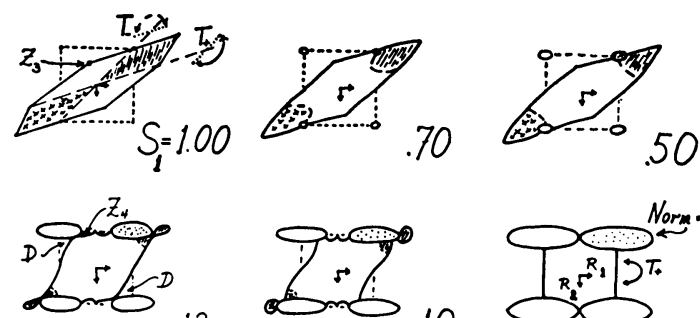

.13
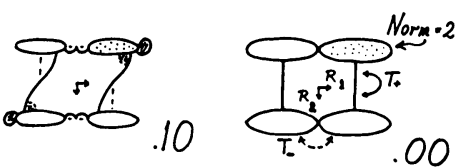

Figure 8. Cross sections of floor for $k=3$. 
17. Special Computations. The cross sections of the floor have been carefully computed for $k=2$ and $k=3$ and are sketched in Figs. 7 and 8. To properly interpret these sketches, we must ignore designations of $T_{+}$and $T_{-}$at first and imagine a symmetric set of sketches for $-h \leqq S_{1} \leqq 0$ according to the symmetry $\left(Z, Z^{\prime}\right) \rightarrow$ $\left(Z^{\prime}, Z\right)$, in effect $T_{+}$(see Section 15 above).

The sketches were set up so as to show the torus structure, i.e., the periodicity of $R_{1}, R_{2}$ modulo 1 . There are two difficulties. First of all, there are pieces of norm 2 which are spindle-shaped, (see Fig. 6 for $k=2$ ), and which occupy several positions which are equivalent modulo $\mathbf{O}$. One such piece is shaded in each diagram for emphasis. (Compare identifying letters on Figs. 6 and 7.) The piece of norm 1 is more difficult because it extends above $S_{1}=h$ and below $S_{1}=-h$. Therefore it is "folded over" into the shaded area of the "parallelogram" type regions. For instance in Fig. 7, for $k=2$, the shaded area transforms like $-\epsilon_{+}^{-1} / Z$, (where $\epsilon_{+}{ }^{-1}=$ $3-2 \cdot 2^{1 / 2}$ ). In Fig. 8, the three-dimensional floor is sketched (at least the upper half) to show the pieces of norm 1 and of norm 2 for $k=3$.
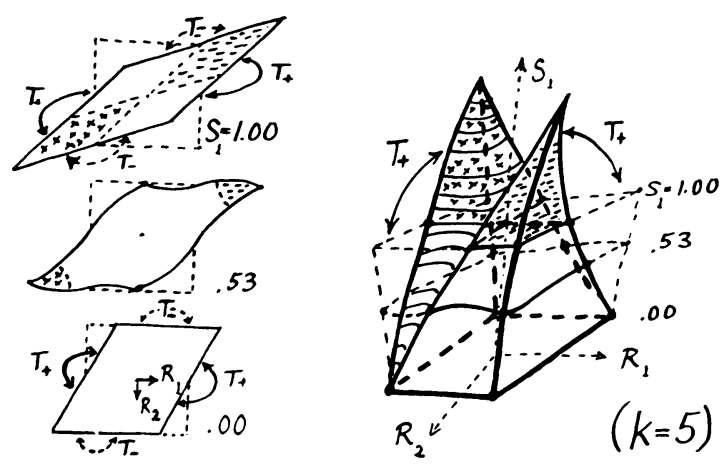

FIGURe 9. Cross sections of floor for $k=5$ and $T_{+}$-symmetrized solid model.

In Fig. 9, we show how the floor would be assembled (for $k=5$ ) once the pieces representing $Z_{*}=-\epsilon_{+}{ }^{-1} / Z$ are folded over for the $T_{+}$symmetry. (The same thing has to be done for Figs. 7,8 and 10 where $k=2,3$ and 6 but the process is too difficult to represent by a drawing!)

For $k=2$, we note in Fig. 7 that the "parallelogram" (of norm 1) twists its way from a position at $S_{1}=0$ to a position at $S_{1}=1$, attaching and detaching itself from the spindle shaped piece (of norm 2). The attachment and detachment points $(D)$ occur at special values which are easy to compute from the output by looking for surfaces $\|\gamma Z+\delta\|=1$ which intersect there (see Remark (j) below). For instance, referring to $k=2$,

$$
\begin{aligned}
& Z_{1}: S_{1}=.31=(\text { really })\left(4-6^{1 / 2}\right) / 5 \\
& Z_{1}: R_{1}=\left(-8+3 \cdot 6^{1 / 2}\right) / 4, R_{2}=\left(3-2 \cdot 6^{1 / 2}\right) / 4 \\
& Z_{1}: S_{2}=\left(-3+2 \cdot 6^{1 / 2}\right) / 4 \\
& Z_{2}: S_{1}=.82=(\text { really }) 6^{1 / 2} / 3 \\
& Z_{2}: R_{1}=\left(2-6^{1 / 2}\right) / 4, R_{2}=\left(-6^{1 / 2}+1\right) / 4 \\
& Z_{2}: S_{2}=\left(-3+2 \cdot 6^{1 / 2}\right) / 4
\end{aligned} \quad(k=2)
$$


Likewise for $k=3$ there only is one special detachment value, at $S_{1}=.13$, but there the ("parallelogram-shaped") piece of norm 1 is split by the (spindle-shaped) piece of norm 2. More strenuously, we find

$$
\begin{aligned}
& Z_{4}: S_{1}=.13=(\text { really! }) \frac{3^{1 / 2}\left(5\left(105^{1 / 2}-9\right)-2\left(30-2 \cdot 105^{1 / 2}\right)^{1 / 2}\right)}{\left(35^{1 / 2}-27^{1 / 2}\right)\left(1 / 2\left(75-7 \cdot 105^{1 / 2}\right)\right)^{1 / 2}} \\
& Z_{4}: R_{1}=1 / 4\left(1 / 2\left(75-7 \cdot 105^{1 / 2}\right)\right)^{1 / 2}, R_{2}=\frac{-21+105^{1 / 2}}{8} \\
& Z_{4}: S_{2}=1 / 4\left(1 / 2\left(15-105^{1 / 2}\right)\right)^{1 / 2}
\end{aligned}
$$

Also at $S_{1}=1$, the configuration is not a rectilinear hexagon. It is of some help however to know $Z_{3}$, namely

$$
\begin{aligned}
& Z_{3}: S_{1}=1 \\
& Z_{3}: R_{1}=\left(2-6^{1 / 2}\right) / 4, R_{2}=\left(-6+6^{1 / 2}\right) / 4 \\
& Z_{3}: S_{2}=\left(-3+2 \cdot 6^{1 / 2}\right) / 4 .
\end{aligned}
$$

The value of $S_{2}$ in (17.1) and (17.2) is conjecturally the minimum for $k=2$. It is rather surprising that it also occurs for (17.4) where $k=3$.

It is also possible to observe an additional symmetry when $\epsilon_{+}=\epsilon_{0}{ }^{2}$, as for $\mathbf{O}\left(2^{1 / 2}\right)$ and $\mathbf{O}\left(5^{1 / 2}\right)$. There the top and bottom faces of $\mathbf{U} \times \mathbf{U} / \mathbf{H}_{\infty}{ }^{+}$or $\mathbf{U} \times \mathbf{U} / \mathbf{H}_{\infty}{ }^{-}$ become reversed. For instance, under (15.5a) and (15.5b) the self-identification is

$$
\begin{aligned}
& T_{+}(X)=X^{\prime}, \quad T_{-}(X)=-X^{\prime} \quad \text { at } S_{1}=0, \\
& T_{+}(X)=\epsilon_{+} X^{\prime}, \quad T_{-}(x)=-\epsilon_{+} X^{\prime} \text { at } S_{1}=h .
\end{aligned}
$$

These would be reversed if we change $Z$ to $\epsilon_{0} \zeta$, i.e., $T_{+}(X)$ at $S_{1}=0$ would become $T_{-}(X)$ at $S=h$, etc. The change in sign occurs because $\epsilon_{0} \epsilon_{0}^{\prime}=-1(!)$.

The symmetry is also manifest in a "plane of symmetry" corresponding to $S_{1}=k^{1 / 2}\left(\epsilon_{0}-1\right) /\left(\epsilon_{0}+1\right)=4-2 \cdot 5^{1 / 2}=.53$ for $k=5$ and $2-2^{1 / 2}=.59$ for $l=2$. The detachment points $Z_{1}$ and $Z_{2}$ in (17.1) and (17.2) are "symmetric" with respect to that plane.

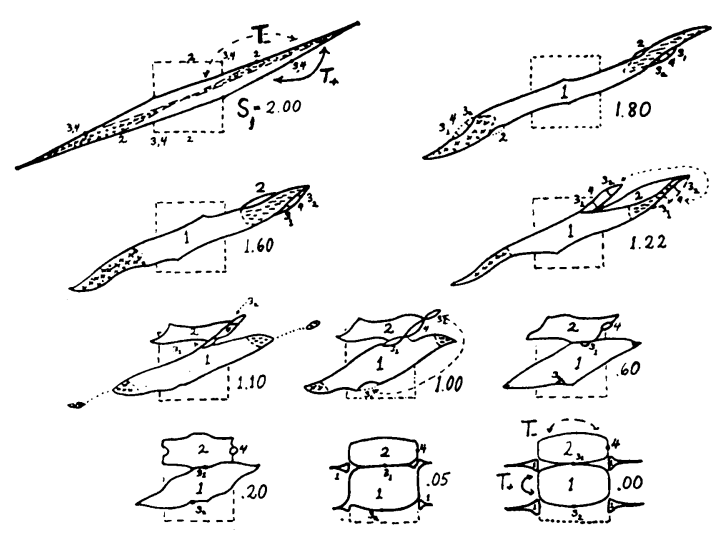

Figure 10. Cross sections of floor for $k=6$. 
In Fig. 10, some cross sections are appended for $k=6$. Here the sketches can make even less pretense to completeness, but they are self-explanatory to the extent they supplement general descriptive information in [12, Section 12]. (Compare Remark (j) below.) There a description was given of how a piece of norm 2 is detached for $S_{1}$ near 1.22. There are also spindle-like pieces of norms 3 and 4 . We show in Fig. 10 how an additional piece of norm 1 is detached. Perhaps the only safe conclusion from Fig. 10 is that in general the pieces will interwind and split one another in much more complicated fashion than imaginable from these illustrations! (Compare [7].)

Summary of Transformations relative to $\mathbf{H}$ (only)

$k=2, \epsilon_{+}=3+2 \cdot 2^{1 / 2}$, (Fig. 7).

Norm 1. $\Sigma_{2}: Z_{*}=-1 / Z$, (shaded area $\left.\Sigma_{23}: Z_{*}=-\epsilon_{+}{ }^{\prime} / Z\right)$.

Norm 2. $\Sigma_{8}: Z_{*}=\left(Z-2^{1 / 2}\right) /\left(2^{1 / 2} Z-1\right)$.

$k=3, \epsilon_{+}=2+3^{1 / 2}$, (Fig. 8 ).

Norm 1. $Z_{*}=-1 / Z$, (shaded area $\left.Z_{*}=-\epsilon_{+}{ }^{\prime} / Z\right)$.

Norm 2. $Z_{*}=\left(Z-3^{1 / 2}\right) /\left(\left(1+3^{1 / 2}\right) Z-1\right)$.

$k=5, \epsilon_{+}=\frac{1}{2}\left(3+5^{1 / 2}\right)$, (Fig. 9).

Norm 1. $Z_{*}=-1 / Z$, (shaded area $\left.Z_{*}=-\epsilon_{+}{ }^{\prime} / Z\right)$.

$k=6, \epsilon_{+}=5+2 \cdot 6^{1 / 2}$, (Fig. 10).

Norm 1. $Z_{*}=-1 / Z$, (shaded area $\left.Z_{*}=-\epsilon_{+}{ }^{\prime} / Z\right)$.

Norm 2. $Z_{*}=\left(Z-6^{1 / 2}\right) /\left(\left(2+6^{1 / 2}\right) Z-1\right)$.

Norm 3. $Z_{*}=(Z-2) /\left(\left(3+6^{1 / 2}\right) Z-1\right)$, (interchanges pieces).

Norm 4. $Z_{*}=(Z-1) /(2 Z-1)$.

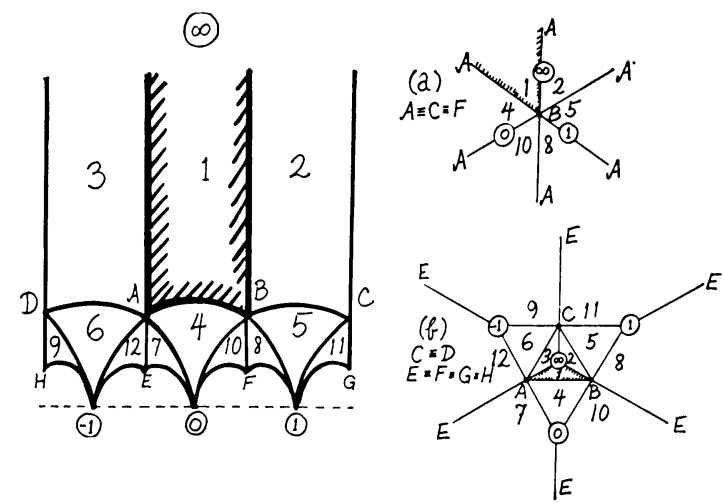

Figure 11. Fundamental domains and principal superdomains, (a) $\bmod 2$ and (b) $\bmod 3$.

18. Conjectured Nontrivial Homology Classes. As a final step in this preliminary report, we call attention to certain 2 -spheres which can be detected in various fundamental domains for $\mathrm{H}^{ \pm}$, which are probably homologously independent. They are generalizations of the 2 -sphere provided by the fundamental domain in the classical modular group, (see [2], and note the shaded region "1" in Fig. 11). 
Start with the floor of $\mathbf{U} \times \mathbf{U} / \mathrm{H}^{+}$as represented for $k=2$ in Fig. 7 (with the $T_{+}$symmetry). First take the two-dimensional set with $S_{2}$ variable and

$$
\begin{aligned}
& S_{1}=0, \\
& R_{1}=0, \\
& R_{2}(\bmod 1) .
\end{aligned}
$$

We assert that this is equivalent to the 2-sphere in a manner analogous to the (2-sphere) region " 1 " in Fig. 11 where the base $A B$ is folded over into itself by $T(Z)=-1 / Z$ and suspended from $\infty$. Here indeed the base $R_{2}(\bmod 1)$ is folded into itself by $T_{+}$and suspended from $\left(S_{2}=\right) \infty$. Likewise, take the two-dimensional set with $S_{2}$ variable and

$$
\begin{aligned}
& S_{1}=0, \\
& R_{2}=0, \\
& R_{1}(\bmod 1) .
\end{aligned}
$$

Here the base $R_{1}(\bmod 1)$ is folded into itself, but not by $T_{+}$. It is folded into itself by the action of the transformation $Z_{*}=-1 / Z$.

Similar configurations can be found in Figs. 8 and 9 . We conjecture these pairs are a two-homology basis for the fundamental domain of $\mathrm{U} \times \mathrm{U} / \mathrm{H}^{+}$or $\mathrm{U} \times \mathrm{U} / \mathrm{H}^{-}$ in each case.

Now, as noted by Gundlach [8], these fundamental domains can never be manifolds. Even if they behave like manifolds at $S_{2}=\infty$, they have singularities at the finite (elliptic) fixed points. This difficulty can be removed by taking a subgroup of $\mathrm{H}^{ \pm}$(which is "too small" to contain these singular fixed points) so we have a super"fundamental domain" consisting of several "building blocks" laid "side by side" in accordance with the quotient group of the subgroup. Of the subgroups cited by Gundlach, two are simple enough to visualize, (to the extent that we can legitimately visualize the original fundamental domain), namely $\mathrm{H}_{2}+$ and $\hat{\mathbf{H}}_{3}+$ as they will be called.

Note in the meantime, that Fig. 11(a), and Fig. 11(b), show how the fundamental domain of the principal congruence subgroup mod 2 and $\bmod 3$ are laid out by use of the fundamental domain " 1 " and images under cosets of the quotient group in the full group. In matrix form, the cosets $(\bmod 2)$ are given by $S_{1}, S_{2}, S_{4}, S_{5}, S_{8}, S_{10}$ while the cosets $(\bmod 3)$ are given by all $S_{1}, \cdots, S_{12}$. The full list is

$$
\begin{array}{rlrl}
S_{1} & =\left(\begin{array}{ll}
1 & 0 \\
0 & 1
\end{array}\right), & S_{2}=\left(\begin{array}{ll}
1 & 1 \\
0 & 1
\end{array}\right), & S_{3}=\left(\begin{array}{rr}
1 & -1 \\
0 & 1
\end{array}\right), \\
S_{4}=\left(\begin{array}{rr}
0 & 1 \\
-1 & 0
\end{array}\right), & S_{5}=\left(\begin{array}{ll}
-1 & 1 \\
-1 & 0
\end{array}\right), & S_{6}=\left(\begin{array}{rr}
1 & 1 \\
-1 & 0
\end{array}\right), \\
S_{7}=\left(\begin{array}{rr}
0 & -1 \\
1 & 1
\end{array}\right), & S_{8}=\left(\begin{array}{ll}
1 & 0 \\
1 & 1
\end{array}\right), & S_{9}=\left(\begin{array}{rr}
-1 & -2 \\
1 & 1
\end{array}\right), \\
S_{10}=\left(\begin{array}{rr}
0 & -1 \\
1 & -1
\end{array}\right), & S_{11}=\left(\begin{array}{rr}
1 & -2 \\
1 & -1
\end{array}\right), & S_{12}=\left(\begin{array}{rr}
-1 & 0 \\
1 & -1
\end{array}\right) .
\end{array}
$$


Next consider $\mathrm{H}_{2}{ }^{+}$, the principal congruence subgroup for $k=2$ of $\mathbf{H}^{+}\left(\bmod 2^{1 / 2}\right)$, i.e., those transformations for which $\Sigma \equiv I$ or $T_{+}\left(\bmod 2^{1 / 2}\right)$. The cosets are in one-to-one correspondence with the corresponding subgroup of the modular group $(\bmod 2)$. This is shown in Table 5 . Here we have six replicas of the fundamental domain arrayed similarly to regions " $1,2,5,8,10,4$ " of Figure 11(a). The transformation $Z_{*}=-1 / Z$ serves to share the floor between regions such as " 1 " and " 4 ." The pieces of norm 2 on the other hand are contracted into themselves by transformations like $Z_{*}=\left(Z-2^{1 / 2}\right) /\left(2^{1 / 2} Z+1\right)$, (which are incidentally $\equiv Z$ $\left.\left(\bmod 2^{1 / 2}\right)\right)$. It is not hard to see that the 2 -sphere in $(18.1)$ is still present, but the 2-sphere in (18.2) is lost since the identification $Z_{*}=-1 / Z$ is not in $\mathrm{H}_{2}{ }^{+}$. Nevertheless another 2-sphere is present in the form of six replicas of set (18.2) pasted together, (as in Fig. 11(a)).

Finally consider $\hat{\mathbf{H}}_{3}+$ the principal congruence subgroup for $k=3$ of $\hat{\mathbf{H}}^{+}(\bmod$ $\left.3^{1 / 2}\right)$. Here we replace $\mathbf{H}^{+}$by $\hat{\mathbf{H}}^{+}$the so called strict Hilbert modular group, defined by using $\epsilon_{0}^{2}=7+4 \cdot 3^{1 / 2}$ instead of $\epsilon_{0}=\epsilon_{+}=2+3^{1 / 2}$. (Equivalently this would mean insisting that in $(2.7) \epsilon$ is a square unit, not just a totally positive unit.) The net effect would be that the fundamental domain of $\mathrm{U} \times \mathrm{U} / \hat{\mathrm{H}}^{+}$would be two replicas of that of $\mathrm{U} \times \mathbf{U} / \mathrm{H}^{+}$, sewed together "in the $S_{1}$ direction." Here Fig. 11(b) would be applicable.

Since further discussion would probably bring us outside of the "surveillance" of the computer, we do not carry the topological investigations any further.

19. Concluding Remarks. (h) The downward direction of the $R_{2}$ axis in Fig. 5 may seem peculiar but it is due to the direction of the paper in the printer!

(i) The point $R_{1}=0, R_{2}=-.50$ seems to have a spurious transformation \#35 associated with it on the print-out. This transformation is not listed since the point is at an intersection of three-dimensional regions of the floor, so other listed transformations (e.g., \#22, \#24, \#26, etc.,) are all valid simultaneously.

(j) The process of locating the critical values (17.1) through (17.4) is illustrated above, (see, especially (12.5)). These four values of $(\gamma, \delta)$ are found by inspection. (Incidentally the correct value in (12.5) was $S_{1}=-1.22 \cdots=-6^{1 / 2} / 2$ although it escaped notice at the time.) Actually in practice we are helped by observations other than $(\gamma, \delta)$; for instance in the case referred to, the output showed that $\operatorname{Im} T(Z)=Y^{\prime}, \operatorname{Im} T\left(Z^{\prime}\right)=Y$, for $T(Z)=\left(\left(1-6^{1 / 2}\right) Z-3\right) /\left(2 Z+\left(1+6^{1 / 2}\right)\right)$. This auxiliary observation is as valuable for computing a point of detachment as the condition $\left\|2 Z+\left(1+6^{1 / 2}\right)\right\|=1$, cited for $(12.5)$.

University of Arizona

Tucson, Arizona 85721

\section{Bibliography}

(Earlier items listed in [6] and [12])

6. H. Coнn, "A numerical survey of the floors of various Hilbert fundamental domains," Math. Comp., v. 19, 1965, pp. 594-605. MR 33 \#4016.

12. H. CoHN, "A numerical study of topological features of certain IIilbert fundamental domains," Math Comp., v. 21, 1967, pp. 76-86. MR 36 \#5081. 
13. H. СонN, "Sphere fibration induced by uniformization of modular group," J. London Math. Soc., v. 43, 1968, pp. 10-20.

Erratum in [6]

p. 603, Table 3. For $k=13$, $\operatorname{Max}|N(\gamma)|=4($ not 3$)$.

Errata in [12]

p. 81, Figure 2. Instead of $S$, read $S_{1}$.

p. 82, Figure 3. Instead of $S=-.80, \operatorname{read} S_{1}=-.40$,

$S=-.60, \quad S_{1}=-.20$,

$S=-.40, \quad S_{1}=0.00$.

pp. 84-85, Figure 4. Instead of $S$, read $S_{1}$. 